\title{
Distributed Mobility Management: a Standards Landscape
}

\author{
Juan Carlos Zúñiga*, Carlos J. Bernardos ${ }^{\dagger}$, Telemaco Melia ${ }^{\ddagger}$, Antonio de la Oliva ${ }^{\dagger}$, Rui Costa ${ }^{\ddagger}$, Alex Reznik ${ }^{\S}$ \\ *InterDigital Canada, Ltd., Montreal, Quebec, Canada, JuanCarlos.Zuniga@InterDigital.com \\ ${ }^{\dagger}$ Universidad Carlos III de Madrid, Leganes, Spain $\{c j b c$, aoliva\}@it.uc3m.es \\ $¥$ Alcatel-Lucent Bell Labs, France, \{telemaco.melia, \\ rui_pedro.ferreira_da_costa\}@alcatel-lucent.com \\ $\S$ InterDigital, King of Prussia, PA, USA, Alex.Reznik@InterDigital.com
}

\begin{abstract}
In this article, we introduce Distributed Mobility Management (DMM) - a new architectural paradigm for evolving mobile IP networks. We discuss the technology trends which are driving a move towards DMM and what the relevant standards development organizations (IETF and 3GPP) are doing to address these new needs. We conclude with a discussion of how 3GPP's Evolved Packet Core (EPC) can evolve towards a DMM-based architecture.
\end{abstract}

\section{INTRODUCTION}

Mobile data traffic has experienced exponential growth over the last few years. We are witnessing the proliferation of smart phone applications that go beyond traditional web traffic to more bandwidth-demanding and delay-sensitive applications such as video. New technologies like HTML5, developed to provide a better experience to mobile users, are also driving an increase in the traffic demand as they make it easier for mobile devices to access content that was traditionally only available to desktop computers.

This increase in demand is having a serious impact on the dimensioning and planning of mobile networks. Specifically, we note that $a$ ) spectrum is limited and expensive, so available bandwidth for the access network cannot be easily increased; and $b$ ) deployed mobile core networks are highly hierarchical and centralized, which introduces serious scalability and reliability issues. Mobile network operators are addressing the first point in several ways, namely, $i$ ) by deploying more spectrum-efficient technologies, such as the 3GPP Long Term Evolution (LTE); ii) by using smaller densely deployed cells, as well as, iii) by selectively offloading traffic from the cellular access to alternative wireless technologies such as WiFi. In order to address the second point, new network architecture approaches that distribute the responsibility of providing connectivity and mobility are needed. Tackling this second point - that is alleviating core network scalability concerns in terms of number of users, required state and traffic load - is the focus of the present article. On one hand, some short-term solutions are being developed as evolution

The research of Carlos J. Bernardos, Telemaco Melia and Antonio de la has been partially funded by the European Community's Seventh Framework Programme (FP7-ICT-2009-5) under grant agreement n. 258053 (MEDIEVAL project). Carlos J. Bernardos has been also partially funded by the Ministry of Science and Innovation of Spain under the QUARTET project (TIN200913992-C02-01) of currently deployed mobile network architectures to provide relief to current traffic problems. On the other hand, long-term alternatives capable of coping with the future expected traffic loads, involving a major redesign of the network architecture, are being researched.

This article presents how current mobility management network architectures are being re-designed towards a more distributed operation that mitigates the problems noted above. These problems are presented in more detail in Section II. Section III presents an overview of the solutions explored by the two main standardization bodies in the field of mobile communications: $\mathrm{IETF}^{1}$, and $3 \mathrm{GPP}^{2}$. Our view of the potential evolution of these solutions is presented in Section IV, where we discuss the future evolution of IP mobility management architectures. Finally, in Section V we make some concluding remarks.

\section{DMM Motivation}

Current packet-based mobile architectures, such as the 3GPP Evolved Packet System (3GPP EPS) and WiMAX, make use of IP as the enabling technology for both voice and data communications. This implies a key-role for IP mobility management in providing the ubiquitous always-on network access service. Even though several applications do not require today the network to provide IP mobility support (meaning IP address continuity), there are still many that do require it (e.g., voice or virtual private networking, to just mention a few of them). Unfortunately, current IP mobility protocols rely on the use of a centralized and hierarchical architecture, which poses several critical issues as explained in more detail next.

Mobility management schemes standardized by IETF for IPv6 networks are extensions to or modifications of the well known Mobile IPv6 protocol (MIPv6) [1], and can be classified into two main families: client-based mobility protocols, and network-based mobility protocols.

Client-based mobility approaches, such as MIPv6 and Dual Stack Mobile IPv6 (DSMIPv6) [2], enable global reachability and session continuity by introducing the Home Agent (HA), an entity located at the home network of the Mobile Node (MN) which anchors the permanent IP address used by the

\footnotetext{
${ }^{1}$ The Internet Engineering Task Force: http://www.ietf.org/

${ }^{2}$ The 3rd Generation Partnership Project: http://www.3gpp.org/
} 
MN, called the Home Address (HoA). The HA is in charge of defending the MN's HoA when the MN is not at home, and redirecting received traffic to the MN's current location. When away from its home network, the MN acquires a temporal IP address from the visited network - called Care-of Address (CoA) - and informs the HA about its current location. An IP bi-directional tunnel between the MN and the HA is then used to redirect traffic to and from the MN.

With network-based mobility management protocols, such as Proxy Mobile IPv6 (PMIPv6) [3], MNs are provided with mobility support without their involvement in mobility management and IP signaling, as the required functionality is relocated from the $\mathrm{MN}$ to the network. In particular, movement detection and signaling operations are performed by a new functional entity called the Mobile Access Gateway (MAG), which usually resides on the Access Router (AR) for the MN. In a Localized Mobility Domain (LMD), which is the area where the network provides mobility support, there are multiple MAGs. The MAG learns through standard terminal operation, such as router and neighbor discovery, or by means of link-layer support about an MN's movement and coordinates routing state updates without any mobilityspecific support from the terminal. The IP prefixes (home network prefixes) used by MNs within an LMD are anchored at an entity called the Local Mobility Anchor (LMA), which plays the role of local home agent of the LMD. Bi-directional tunnels between the LMA and the MAGs are set up, so the $\mathrm{MN}$ is enabled to keep the originally assigned IP address despite its location changes within the localized mobility domain. Through the intervention of the local mobility anchor, packets addressed to the $\mathrm{MN}$ are tunneled to the appropriate mobile access gateway within the LMD, hence making the mobile node oblivious to its own mobility. The 3GPP also supports a variant of network-based IP mobility which uses the General Packet Radio Service (GPRS) tunneling protocol protocol instead of PMIPv6, with the Packet Gateway (PGW) playing the role of the LMA in the EPS and the GGSN playing the role of the LMA in the legacy UMTS/GPRS network.

Whatever flavor of IP mobility a modern day Mobile Network Operator (MNO) chooses to deploy, a constant feature of the operator's architecture will be the presence of a central entity (HA/LMA/PGW/GGSN) which anchors the IP address used by the mobile node. This centralized function is in charge of coordinating the mobility management; in the 3GPP this is done in conjunction with a control function called the Mobility Management Entity (MME). As a result, these centralized mobility anchors are burdened with data forwarding and control mechanisms for a huge number of users. Fig. 1 summarizes the operation of the IETF Mobile IPv6, Proxy Mobile IPv6 protocols, as well as the GPRS Tunneling Protocol (GTP), which is one of the network-based mobility variants adopted by the 3GPP. Table I shows, for the main mobility protocols and architectures, the equivalence between the principal mobility roles and the logical entities playing them.

While this centralized way of addressing mobility management has been fully developed by the Mobile IP protocol family and its many extensions, it brings several limitations

\begin{tabular}{|c|c|c|c|c|}
\hline & MIPv6 & Proxy MIPv6 & GPRS \& UMTS & EPS \\
\hline Mobility anchor & HA & LMA & GGSN & PGW \\
\hline Signaling agent & MN & MAG & SGSN & SGW/ePDG/eNB \\
\hline
\end{tabular}

TABLE I

EQUIVALENCE BETWEEN MAIN MOBILITY ROLES AND LOGICAL ENTITIES.

that have been identified in [4]:

- Sub-optimal routing. Since the (home) address used by a mobile node is anchored at the home link, traffic always traverses the central anchor, leading to paths that are, in general, longer than the direct one between the mobile node and its communication peer. This is exacerbated with the current trend in which content providers push their data to the edge of the network, as close as possible to the users, as for example deploying Content Delivery Networks (CDNs). With centralized mobility management approaches, user traffic will always need to go first to the home network and then to the actual content source, sometimes adding unnecessary delay and wasting operator's resources.

- Scalability problems. Existing mobile networks have to be dimensioned to support all the traffic traversing the central anchors. This poses several scalability and network design problems, as central mobility anchors need to have enough processing and routing capabilities to be able to deal with all the users' traffic simultaneously. Additionally, the entire operator's network needs to be dimensioned to be able to cope with all the users' traffic.

- Reliability. Centralized solutions share the problem of being more prone to reliability problems, as the central entity is potentially a single point of failure.

In order to address these issues, a new architectural paradigm, the so-called Distributed Mobility Management (DMM), is being explored by both research and standards communities. DMM introduces the concept of a flatter system architecture, in which mobility anchors are placed closer to the user, distributing the control and data infrastructures among the entities located at the edge of the access network. Critically, DMM introduces the ability for a mobile node to move between mobility anchors, something that is not possible with any of the present centralized approaches. Additionally, it is worth noting that by removing all the difficulties posed by the deployment of a centralized anchoring and mobility approach, adoption of DMM approaches is expected to be easier.

\section{DMM IN STANDARDS}

In this section we provide an overview of current DMM or DMM-like initiatives in the IETF and 3GPP standards organizations.

\section{A. DMM in the Internet Engineering Task Force (IETF)}

The IETF is at the moment the main driver standardizing solutions for Distributed Mobility Management. The DMM working group ${ }^{3}$ was chartered in March 2012 to address

\footnotetext{
${ }^{3}$ http://datatracker.ietf.org/wg/dmm/
} 

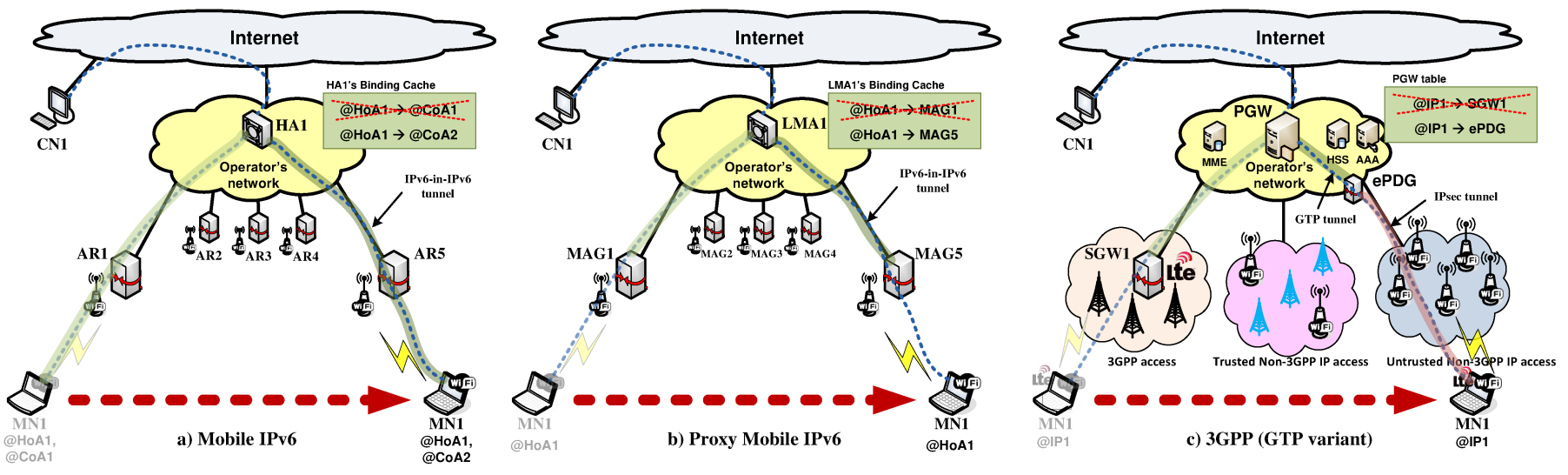

Fig. 1. Overview of the MIPv6, PMIPv6 and GTP protocols.

the emerging need of mobile operators to evolve existing IP mobility solutions towards supporting a distributed anchoring model. The IETF has first identified the requirements that an IP distributed mobility management solution should meet [4], and it is currently analyzing existing practices for the deployment of IP mobility solutions in a DMM environment [5]. The goal of this analysis is to identify what can be achieved with existing mobility solutions and which functions are missing in order to meet the identified DMM requirements.

In terms of solution space, three main classes of solutions can be identified: i) client-based, ii) network-based, and iii) routing-based approaches (see Fig. 2 for operational examples of each of them). In the case of client-based mobility, existing proposals aim at deploying multiple home agents at the edge of the access network thus distributing the anchoring. The basic concept is that a mobile node no longer uses a single IP address anchored at a central home agent, but it configures and uses an additional IP address at each visited access network. The mobile node uses the locally-anchored address to start new communications, while maintaining the reachability for those IP addresses that are still in use by active communications. This requires the mobile node to bind each of the active (home) addresses with the locally-anchored address currently in use, which is actually playing the role of care-of address in these bindings. Session continuity is guaranteed by the use of bi-directional tunnels between the MN and each one of the home agents anchoring in-use addresses, as shown in Fig. 2 a). This deployment model, proposed for example in [6] and [7], does not require changes on the protocol behavior of the network entities. However, it requires extensions and additional intelligence on the mobile node side, as it has to manage multiple addresses simultaneously, select the right one to use for each communication, keep track of those addresses which need mobility support, and perform the required maintenance operations (i.e., binding signaling and tunneling). Additionally, non-locally-anchored traffic experiences sub-optimal routing. Consider the example in Fig. 2 a); here the mobile node MN1 initially attaches to the distributed anchor HA/AR1 and configures the IPv6 address HoA1 to communicate with a correspondent node $\mathrm{CN} 1$. If MN1 moves to HA/AR5, a new locally-anchored IPv6 address is configured (H०A2) and used for new communications (for example with $\mathrm{CN} 2$ ). The continuity of the session with $\mathrm{CN} 1$ is provided by a tunnel set-up between the mobile node and HA/AR1.

With respect to the network-based solutions, two sub families can be identified: $i$ ) solutions with a fully distributed model, and ii) solutions with a partially distributed model. The distinction between fully and partially distributed approaches has to do with whether the control plane and the data plane are tightly coupled or not. In the fully distributed model, mobility anchors are moved to the edge of the access network and they manage both control and data plane. For instance, in a fully distributed model and using PMIPv6 terminology, each access router implements both LMA and MAG functions and for each user the access router could behave as a local mobility anchor (thus anchoring and routing the local traffic for a given user) or as a mobile access gateway (thus receiving the tunneled traffic from the virtual home link of the given user). If we consider a partially distributed model, data plane and control plane are separated and only the data plane is distributed. In this sense, the operations are similar to 3GPP networks where the control plane is managed by the MME and the data plane by the SGW and PGW. An example of the operation of a generic network-based DMM solution is shown in Fig. 2 b).

The split of the control plane and data plane allows the mobility anchors to optimally route the data traffic while relying on a single central entity to retrieve the localization of the connected mobile devices, namely the tuple \{current mobility anchor router, mobile node identifier $\}$. There are proposals to extend the PMIPv6 protocol to achieve this either by maintaining legacy with current PMIPv6 deployments or by proposing new extensions and changing the way PMIPv6 functions are implemented. Among the ones of the first category, [8] proposes implementing local routing at the MAG. In fact, the PMIPv6 binding signaling exchange is extended to allow the MAG to defend a pool of IP addresses thus routing traffic directly through the Internet. To access the operator services the mobile node can still use the IP address anchored at the LMA. This however requires the management of several IP address at the MN and the selection of a specific IP address for a specific service. Alternatively, [9] introduces the logical entity of the central mobility database to maintain users' localization information and to allow the setup of ondemand tunneling when a specific service requires seamless 

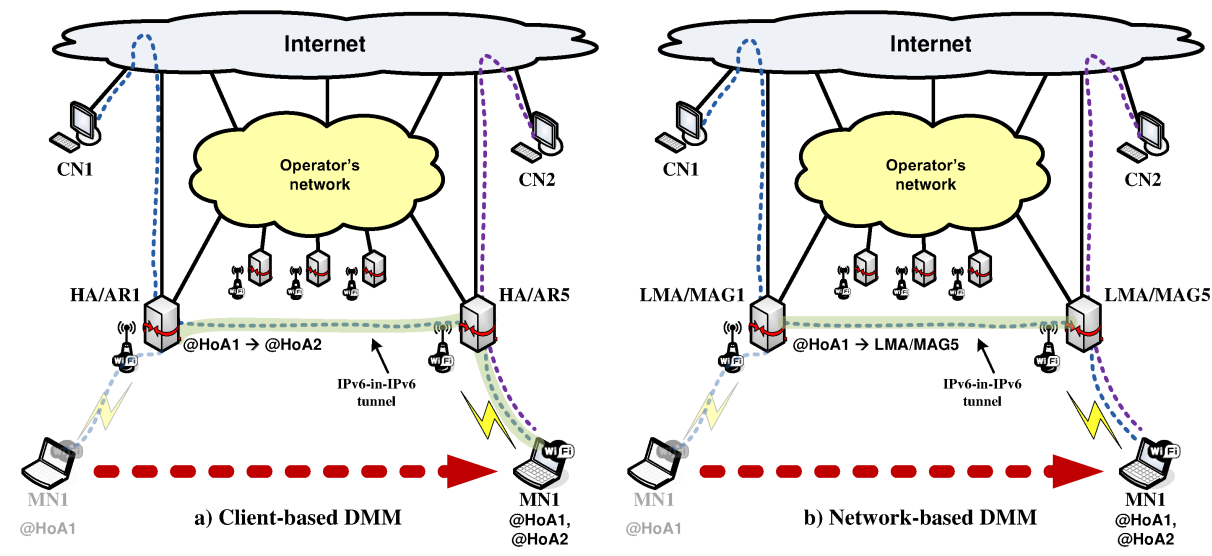

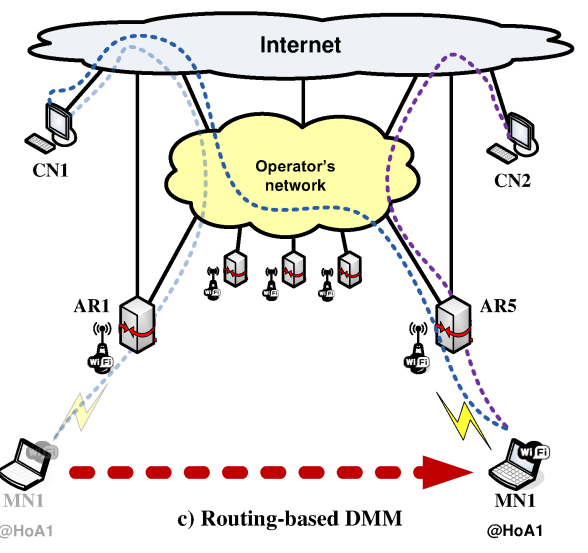

Fig. 2. Client-, network- and routing-based DMM approaches.

mobility support.

It is worth highlighting that network-based approaches pose more challenges than host-based ones, as the mobile terminal needs to appropriately handle different IP addresses in both cases, but this is harder to achieve without some support from the host.

Last, routing-based proposals, such as [10], follow a completely different approach. In this case, when the mobile node attaches to an access router, it obtains an IP address that is then internally advertised within the domain using an intra-domain protocol (e.g., IBGP). When the node moves and attaches to a different access router, the access router finds out the address assigned to the mobile node during the authentication phase, and then proceeds to update its routability via routing updates. In this way, the reachability of the node is ensured while moving within the domain. This approach, however, has some limitations in terms of handover latency (limited by the intra-domain routing convergence) and scalability (i.e., storms of routing updates). An example of this type of solution is shown in Fig. 2 c).

In addition to these three main DMM solution classes, the IETF is also looking at how to distribute the anchoring and mobility support with other approaches, such as Mobile LISP (Locator/Identifier Separation Protocol) and ILNP (IdentifierLocator Network Protocol). However, in this article we have limited the scope of our analysis to DMM evolutionary extensions of approaches exhibiting a proven record in industry and some deployment experience, therefore not covering more disruptive and/or clean slate architectures.

Overall, the IETF DMM group is still in early stages of standardization and it is difficult to draw a full picture of what eventually will become the proposed solution. It is however clear that there is strong interest to address the current issues, especially when new services (e.g., distributed caching for multimedia content or $\mathrm{CDN}$ ) representing renewed business revenues for the mobile network operators require a paradigm shift in the way mobility support is provided today.

\section{B. DMM in the Third Generation Partnership Project (3GPP)}

Although no DMM-specific efforts are on-going in 3GPP, several developments indicate a trend towards a more flex- ible and dynamic mobility management. In this section, we summarize these developments. Later, in Section IV, we show how these solutions can be built upon to introduce full-blown DMM concepts into 3GPP.

Our discussion focuses on the evolved packet core [11] and we begin by discussing the two architectural entities that are key to IP traffic management: the Serving Gateway (SGW) and the Packet Data Network Gateway (PGW). The SGW is the gateway function in the $3 \mathrm{GPP}$ which terminates the interface towards the radio access network (E-UTRAN in 3GPP lingo), which means the SGW is the point in the network beyond which the mobile node ${ }^{4}$ can maintain connection only at IP layer or above. The SGW is also in charge of belowIP mobility between base stations in a single E-UTRAN. However, the SGW does not provide IP mobility anchoring capabilities and so, while the SGW is often located at the edge of the core network, it cannot support DMM-like functionality.

The PGW is the anchor node for IP services, including IP based mobility, IP address management, and other operator services such as data traffic monitoring for the purposes of QoS control, charging, etc. While an operator may maintain multiple PGWs, these gateway functions are used to provide a particular service to the whole operator network, or at least a significant portion of it. For example, an USA-based operator may maintain separate PGWs for its New England operations and its California operations, but it would not do so for each neighborhood in the city of Boston. As such, IP mobility between PGWs is not supported. In fact, the UE cannot even keep the same IP address if it changes PGWs. In the event that the UE does move from one PGW to another, it will experience an interruption of data services. Of course, given the large-scale coverage of PGWs, this is unlikely and thus lack of mobility support across PGWs is not a major concern for operators.

However, the fact that a central gateway node anchors a significant amount of traffic for network's users in a very large geographical area does raise other serious issues, as introduced in Section II. Two issues are particularly acute: $i$ ) the sheer amount of traffic "hitting" the PGW; and ii) the often highly

\footnotetext{
${ }^{4}$ In 3GPP, the mobile node is called User Equipment (UE). In this article we use both terms, UE and MN, to refer to a mobile node.
} 
sub-optimal routes which the need to anchor traffic at the PGW forces. It would appear that one way to address this problem would simply be to allow PGWs to cover much smaller areas and/or become much more service specific. However, this would necessitate support of one key feature currently not supported by the PGW: inter-PGW mobility. In fact, as we shall describe in detail shortly, this is precisely what DMM provides, and thus we believe that DMM offers the right longterm paradigm for the Evolved Packet Core (EPC) evolution.

Nevertheless, a full DMM approach is still somewhat far away in time. In the near term though, 3GPP does provide two approaches designed to relieve the loading introduced by IP traffic into the 3GPP core: Selected IP Traffic Offload (SIPTO) and Local IP Access (LIPA) [11]. SIPTO enables an operator to offload certain types of traffic at a network node close to the UE's point of attachment to the access network, by selecting a set of gateways (SGW and $\mathrm{PGW}$ ) that are geographically or topologically close to the UE's point of attachment. The upside to the operator is lower load on its network. However, mobility support for SIPTO traffic can be rather limited, and offloaded traffic cannot access operator services (as they are only available in the private operator's network). Thus, the operator must be very careful in selecting which traffic to offload.

LIPA, on the other hand, enables an IP-capable UE connected via a Home eNode B (HeNB) to access other IP capable entities in the same residential/enterprise IP network without the user plane traversing the mobile operator's network core. In order to achieve this, a Local Gateway (L-GW) is used, which implements a subset of the PGW functionality. LIPA is established by the UE requesting a new Packet Data Network (PDN) connection to an Access Point Name (APN) for which LIPA is permitted, and the network selecting the local GW associated with the HeNB and enabling a direct user plane path between the local GW and the HeNB.

Like SIPTO, mobility support for traffic offloaded using LIPA is very limited. However, a 3GPP work item on LIPA Mobility and SIPTO at the local Network (LIMONET) [12] is currently studying how to provide SIPTO and LIPA mechanisms with some additional, but still limited, mobility support. In general and without going into fine details, LIPA mobility support is limited to handovers between HeNBs that are managed by the same L-GW (i.e., mobility within the local domain), while seamless SIPTO mobility is still limited to the case where the SGW/PGW is at or above Radio Access Network (RAN) level. Seamless mobility at the local network is still not considered in SIPTO. Therefore, although SIPTO and LIPA allow offloading traffic from the network core in ways that appear similar to the DMM approaches, even with LIMONET these provide only localized mobility support; and when the UE moves outside its local area, packet data connections have to be deactivated and re-activated.

Another important aspect of the present 3GPP approach to mobility management is the role of the Mobility Management Entity (MME). While we do not provide a full overview of this critical architectural function in 3GPP, it is worth noting some of the key functions that the MME supports [11]:

- UE reachability. This includes maintaining information

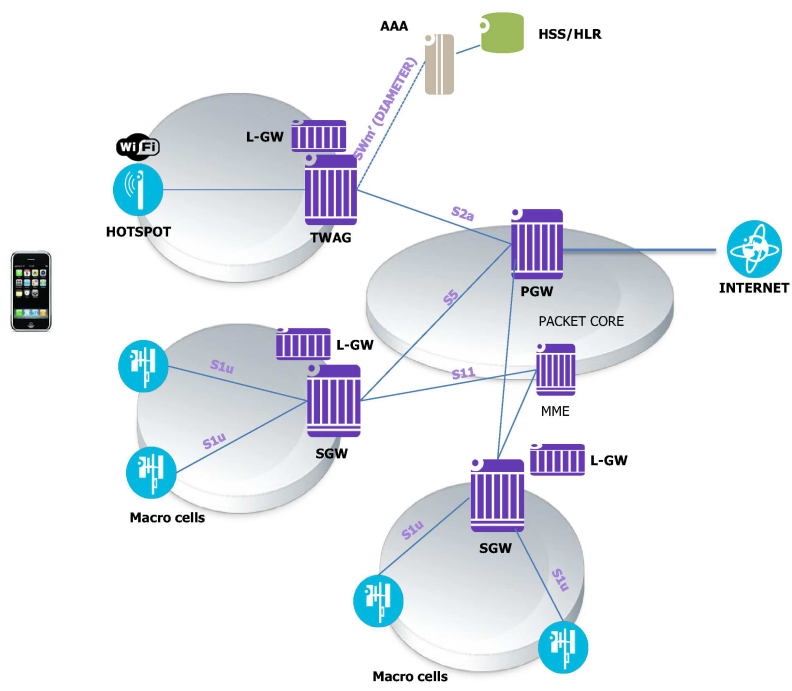

Fig. 3. Network evolution

about the UE state and location of connection to the network as well as idle mode procedures such as paging.

- Selection of the gateway (PGW and SGW) which the UE should be using.

- Management of bearers associated with the UE.

How the MME evolves and changes with the introduction of DMM concepts into the 3GPP network is a subject worthy of a separate paper. However, it should be clear that the role of MME in such evolved networks is an important consideration. For instance, the MME may play a role in the selection of the "distributed" anchor.

\section{Possible Evolution}

As stated in Section III-B, 3GPP is currently standardizing methods to deploy local GWs addressing enterprise scenarios (e.g., LIPA) and more traditional cellular deployments (e.g., SIPTO above the RAN). Considering that LIPA is a 4G-only solution and that SIPTO Release 10 has been specified for both $3 \mathrm{G}$ and $4 \mathrm{G}$ networks, we focus on the $4 \mathrm{G}$ network (EPC), deliberately leaving aside considerations on the integration of legacy networks.

There are two main drivers of distributed anchoring GWs for cellular networks. The first is geographical. In some countries mobile network operators are expected to cover a geographically large territory, hence requiring deployment of a large number of PGWs with a certain degree of locality. The second driver is the need to support a new set of services which require a network of service-anchoring gateways. Among these, multimedia content distribution is perceived as a particularly acute problem facing mobile network operators. A promising approach to address this issue is via distribution of caching systems as overlay networks (e.g., CDNs); however such a distributed caching requires a distributed anchoring solution to go along with it. As these new services emerge as important new sources of revenue for the mobile operators, the operators are ever more like to invest in upgrades of their network infrastructure that support these new revenue sources. 
A distributed mobility management approach is widely believed to be the likely upgrade path. As we shall see, it provides a means to distribute a network gradually and partially, giving the operator the ability to easily evolve from a centralized architecture to a distributed one - and distributing mobility anchoring only to the extent it needs to.

SIPTO above the RAN can be considered the first step. As depicted in Fig. 3 the distribution of the L-GW on top of the SGW assigns users to a closer gateway, enabling a better response in terms of round trip delays and localization of services. The main drawback of current solutions is that when the UE moves from one region to another the local GW may change, and there is no support of seamless mobility between local GWs. This may not be an issue for applications that can survive an IP address change (e.g., for a given access point name the UE has to re-establish the PDN connection to the new GW and therefore change its IP address) but it could impact the ongoing sessions such as a Voice over IP (VoIP) call. One strategy to solve this issue could be to enable data forwarding between the old GW and the new GW, but in terms of 3GPP semantics this is not possible since the UE would need to configure two IP addresses for the same access point name on two different PGWs (e.g., an APN implies one PDN connection hence one IP address). There are solutions that permit the use of an APN simultaneously on both cellular and WiFi, like the seamless Network-Based IP Flow Mobility (NB-IFOM), although they have not yet been fully described in the specifications. Also, since Release 9, it is possible to enable Multiple PDN connections to the same APN for PMIPv6-based interfaces (MUPSAP). This, however, is limited to PMIPv6-based interfaces. Moreover, as of today only a few operators are deploying the S5 interface (i.e., the one providing user plane tunneling between the SGW and the PGW) based on PMIPv6. GTP on the other hand, inherently supports this functionality.

The next step would be to enable the selection of L-GW also for WiFi networks, assuming the mobile network operator is deploying the so-called trusted WiFi access (i.e., S2a-based Mobility Over GTP - SaMOG). Some past proposals do develop SIPTO for WiFi, but they are primarily targeting traffic offload and do not have enough support from manufacturers and service providers. The use of distributed caching at anchors closer to the UE could renew the need for standardizing such functionality.

Now that we have introduced multiple local PGWs in the cellular network and converged the LIPA and SIPTO functions under the same framework, these L-GWs can be used to support a full-fledged DMM framework. Full inter-GW mobility is enabled using techniques such as those presently under consideration by IETF, while LIPA and SIPTO become features that are enabled by the overall framework. One example of how this is done can be found in [13].

\section{CONCLUSION}

The challenges facing mobile network architectures in the application and service centered future are indeed tremendous, as data demands of mobile users are already stressing the existing network. Yet, we believe that a flexible approach to network architectures, combined with an equally flexible spectrum and coverage strategy can go a long way towards addressing these challenges. Indeed, architectural flexibility will allow network operators to offer the "right network" to the "right services." However, to be successful, a "flexible network" technology must be an evolution of existing networks. The alternative would require operators to abandon billions of dollars of existing investment for what is likely to be billions of dollars to deploy a brand new network - a proposition that is not palatable in practical terms.

We believe DMM offers the right solution. As an architectural paradigm, it is designed to be flexible and distributed "from the core," and it builds and evolves from existing IETF and 3GPP mobility protocols. Additionally, it can be introduced into $3 \mathrm{GPP}$ in a gradual and additive fashion, either complementing or replacing existing functions, depending on the operators needs. Moreover, it evolves those features of the 3GPP systems, which are already being deployed as spot solutions to the bandwidth crunch. Accordingly, we encourage the community at large to follow the development of this technology closely.

\section{REFERENCES}

[1] C. Perkins, D. Johnson, and J. Arkko, "Mobility Support in IPv6," RFC 6275 (Proposed Standard), Internet Engineering Task Force, July 2011.

[2] H. Soliman, "Mobile IPv6 Support for Dual Stack Hosts and Routers," RFC 5555 (Proposed Standard), Internet Engineering Task Force, June 2009.

[3] S. Gundavelli, K. Leung, V. Devarapalli, K. Chowdhury, and B. Patil, "Proxy Mobile IPv6," RFC 5213 (Proposed Standard), Internet Engineering Task Force, Aug. 2008.

[4] H. Chan, "Requirements of distributed mobility management," InternetDraft (work in progress), Nov. 2012.

[5] J. C. Zuniga, C. J. Bernardos, T. Melia, and C. Perkins, "Mobility Practices and DMM Gap Analysis," Internet-Draft (work in progress), Dec. 2012.

[6] B. Sarikaya, "Distributed Mobile IPv6," Internet-Draft (work in progress), Feb. 2012.

[7] F. Giust, A. de la Oliva, and C. J. Bernardos, "Flat Access and Mobility Architecture: an IPv6 Distributed Client Mobility Management Solution," in 3rd IEEE International Workshop on Mobility Management in the Networks of the Future World (Mobiworld 2011), in conjunction with IEEE INFOCOM 2011, Apr. 2011.

[8] J. Korhonen and T. Savolainen, "Local Prefix Lifetime Management for Proxy Mobile IPv6," Internet-Draft (work in progress), Mar. 2012.

[9] C. J. Bernardos, A. de la Oliva, F. Giust, T. Melia, and R. Costa, "A PMIPv6-based solution for Distributed Mobility Management," InternetDraft (work in progress), Mar. 2012.

[10] P. McCann, "Authentication and Mobility Management in a Flat Architecture," Internet-Draft (work in progress), Mar. 2012.

[11] 3GPP, "General Packet Radio Service (GPRS) enhancements for Evolved Universal Terrestrial Radio Access Network (E-UTRAN) access," 3rd Generation Partnership Project (3GPP), TS 23.401, Sept. 2011.

[12] — "LIPA Mobility and SIPTO at the Local Network," 3rd Generation Partnership Project (3GPP), TR 23.859, July 2011.

[13] C. J. Bernardos, J. C. Zuniga, and A. Reznik, "Towards Flat and Distributed Mobility Management: a 3GPP Evolved Network Design," in First ICC Workshop on Telecommunication Standards, 2012. 


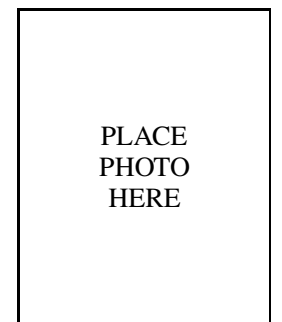

Juan Carlos Zuniga is a Principal Engineer at InterDigital, currently leading the standardization activities in the area of IP mobility, heterogeneous networks and distributed content management. $\mathrm{He}$ has actively contributed and held leadership roles in different standards fora, such as IEEE 802, IETF, and 3GPP. He has been with InterDigital since 2001. Previously he worked with Harris Communications in Canada, Nortel Networks in the UK and $\mathrm{Kb} / \mathrm{Tel}$ in Mexico. He received his engineering degree from the UNAM, Mexico, and his M.Sc. and DIC from the Imperial College, London. He is an inventor of more than 25 granted U.S. patents and 70 published applications. He has been granted several recognitions like the BoD Chairmans Award to the most outstanding patent application, and the British Council Fellowship.

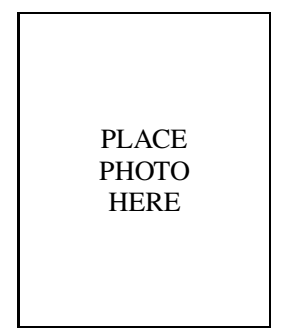

Carlos J. Bernardos received a telecommunication engineering degree in 2003 and a Ph.D. in telematics in 2006, both from UC3M, where currently he works as an associate professor. From 2003 to 2008 he worked at UC3M as a research and teaching assistant. His current work focuses on IP-based mobile communication protocols and vehicular networks. His Ph.D. thesis focused on route optimization for mobile networks in IPv6 heterogeneous environments. He has actively contributed and held leadership roles in the IETF. He has published over 50 scientific papers in prestigious international journals and conferences and has served as guest editor of IEEE Network.

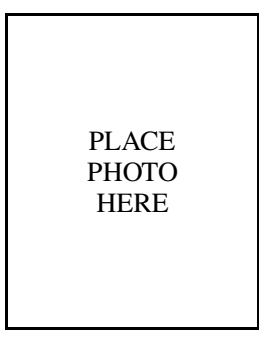

multimedia platforms.
Telemaco Melia received his Informatics Engineering degree in 2002 from the Polytechnic of Turin, Italy and his $\mathrm{PhD}$ in Mobile Communications from University of Goettingen in April 2007. Until December 2007 he worked at NEC Europe Ltd. in the Mobile Internet Group focusing on IP mobility support across heterogeneous. In September 2008 he joined Alcatel-Lucent Bell Labs working on the 3GPP Evolved Packet Core and IETF standardization bodies. His main research interests include wireless networking, next generation networks and

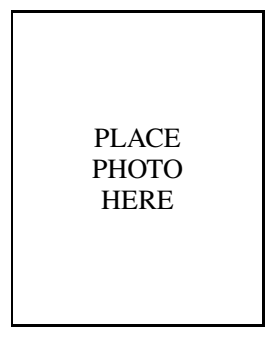

Antonio de la Oliva received a Telecommunication Engineering degree in 2004, and a $\mathrm{PhD}$ in Telematics in 2008, both from the University Carlos III of Madrid (UC3M), where he worked as a lecturer and researcher since 2005. His research is focused to mobility in heterogeneous networks and wireless systems. He has published over 20 scientific papers in prestigious international journals and conferences, and he is also an active contributor of the IEEE 802.21 where he has served as Vice-chair of IEEE $802.21 b$.

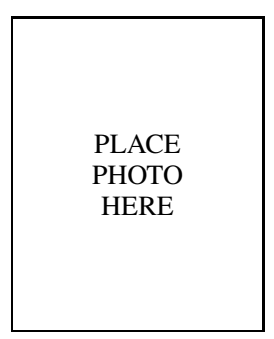

Rui Costa took his MsC. in Computers and Telematics in 2008, from the University of Aveiro, Portugal. During the spring-summer semester of 2007 he was an intern for the IVC group of NEC Deutschland, in Heidelberg, Germany. He currently is a PhD. student with Alcatel-Lucent Bell Labs France. His interests are focused on DMM, PMIP and mobile, vehicular and heterogeneous networks.

\begin{tabular}{|c|}
\hline \\
PLACE \\
PHOTO \\
HERE \\
\end{tabular}

Alex Reznik is a Senior Principal Engineer at InterDigital, currently leading the companys research and system design activities in the area of IP mobility and heterogeneous networks. Since joining InterDigital in 1999, he has been involved in a wide range of projects, including leadership of $3 \mathrm{G}$ modem ASIC architecture, design of advanced wireless security systems, and coordination of standards strategy in the cognitive networks space. He earned his B.S.E.E. Summa Cum Laude from The Cooper Union, S.M. in Electrical Engineering and Computer Science from the Massachusetts Institute of Technology, and Ph.D. in Electrical Engineering from Princeton University. He holds a visiting faculty appointment at WINLAB, Rutgers University, where he collaborates on research in cognitive radio, wireless security, and future mobile Internet. He is an inventor of 70 granted U.S. patents, and has been awarded numerous awards for Innovation at InterDigital 